\title{
Valoración cognitiva y funcional del paciente psicogeriátrico en una unidad psiquiátrica de corta estancia
}

\author{
Ricardo López-Bernués ${ }^{1}$, Yolanda Marcén ${ }^{2}$ y Ana I. Sieso ${ }^{1}$ \\ ${ }^{1}$ Servicio Aragonés de Salud (España); ${ }^{2}$ Universidad de Zaragoza (España)
}

\begin{abstract}
Las alteraciones psiquiátricas presentes en las demencias (deterioro cognitivo) conforman los síntomas psicológicos y conductuales en las demencias (SPCD), presentes en casi la totalidad de estos pacientes y motivo fundamental de ingreso hospitalario de los mismos. Metodología: Se analizó el deterioro cognitivo (MEC de Lobo, test de Pfeiffer y fluencia verbal semántica) y las capacidades funcionales (test de Barthel) de los pacientes mayores de 65 años ingresados en una unidad psiquiátrica de corta estancia durante 3 meses relacionando éstos con los SPCD (Inventario Neuropsiquiátrico de Cumming, [NPI]) y las diferentes variables socio-demográficas analizadas en el estudio. Resultados: La mitad de los 18 pacientes del estudio tenía algún tipo de sintomatología depresiva y entre el 22-44\% presentaban indicios de deterioro cognitivo, según el test de cribaje utilizado. Discusión/Conclusiones: El test que mide las alteraciones psiquiátricas (NPI) está directamente relacionado con el grado de discapacidad para las AVD's y éste a su vez con los días de estancia hospitalaria. Los diferentes porcentajes de afectación del deterioro cognitivo según el test administrado muestran la importancia de la elección del test según la población a estudio y el tipo de análisis realizado.
\end{abstract}

Palabras clave: Deterioro cognitivo, personas mayores, alteraciones psiquiátricas, hospitalización.

Cognitive and functional apraisal in a psychogeriatric patient in a short stay psychiatric unit. Psychiatric disorders are present in dementia (Cognitive damage) and they are part of the psychological and behavioral dementias (SPCD), which are present in almost every patient and a very important cause for psychogeriatric patients'admission. Methodology: Cognitive damage was analysed (MEC de Lobo, Pfeiffer test and semantic and verbal fluency) and functional abilities in elderly inpatients, who were older than 65 years old, were also analysed (Barthel test) for 3 months. Relating these to the different sociodemographic variables. Results: Half of the 18 patients who were analyzed and studied, had any type of depressive symptoms and between $22-44 \%$ showed some signs of cognitive damage according to the test used to sieve. Discussion/Conclusions: Psychiatric disorders measured with NPI are directly related to the disability degree for AVD's and at the same time, this is related to the admission span. The different percentages which affect cognitive damage according to the test used, shows the importance of the correct test's choice depending on the studied population and the type of analysis accomplished.

Keywords: Cognitive damage, elderly people, psychiatric disorders, admission.

Correspondencia: Ricardo López Bernués. Servicio Aragonés de Salud. Avd. Martínez de Velasco, 24, 6B. C.P.: 22004. Huesca (España). E-mail: rilober@ hotmail.com 
Durante la década de los noventa se reactiva el interés científico y sanitario por la sintomatología no cognoscitiva de las demencias. La elevada prevalencia de estos síntomas y las consecuencias en los pacientes y sus familiares-cuidadores propició que en el año 2000 la Asociación Internacional de Psicogeriatría (1998) concluyera que: “el término trastornos conductuales debería ser reemplazado por el de síntomas psicológicos y conductuales en las demencias (SCPD), definido de este modo: síntomas de alteración de la percepción, del contenido del pensamiento, el estado de ánimo o la conducta que a menudo se presentan en pacientes con demencia".

Los SCPD son altamente prevalentes y casi el $100 \%$ de los pacientes con demencia pueden desarrollar algún tipo de estos síntomas a lo largo de la enfermedad. Los más frecuentes e incapacitantes son la depresión, los síntomas psicóticos (alucinaciones, ideas delirantes y falsos reconocimientos), la agitación/agresividad, el vagabundeo y las reacciones catastróficas (Ballard, O’Brien, James y Swan, 2001).

\section{El paciente psicogeriátrico en el hospital general}

Según Blazer (2000), las camas de los hospitales generales están ocupadas en un $60 \%$ por pacientes mayores de 65 años. Los pacientes mayores de 65 años ingresados en un hospital general tienen una alta comorbilidad con las enfermedades mentales incluyendo un $40 \%$ de demencia, un 53\% de depresión y hasta un $61 \%$ de los cuadros confusional es tipo delirium dependiendo de la bibliografía consultada.

O’Keeffe y Lavan (1997) mostraron que los cuidados requeridos por los pacientes ancianos en los hospitales generales se complican por la existencia de patología psiquiátrica comórbida, asociándose ésta a un impacto negativo sobre la evolución clínica del paciente e influyendo en un aumento de la mortalidad, la estancia hospitalaria y las tasas de institucionalización.

\section{Paciente psicogeriátrico en unidad de corta estancia (UCE) psiquiátricas}

Existen pocos estudios que aborden el tema del paciente psicogeriátrico en el ámbito de los servicios de psiquiatría de un hospital general.

Según un estudio descriptivo descrito por Subirá y Cardoner (2010), sobre el total de pacientes mayores de 65 años ingresados en el servicio de psiquiatría del Hospital Universitario de Bellvitge, en el período comprendido entre 1988 y 1994, aproximadamente la mitad (50.9\%) de los pacientes mayores hospitalizados presentaban un Trastorno Depresivo Mayor, un 11\% un Trastorno Mental Orgánico, un 10.3\% un Trastorno Bipolar, un 9.9\% Distimia, un 5.7\% un Trastorno de Ansiedad Generalizada, un $4.6 \%$ un Trastorno Adaptativo, un $3.2 \%$ un Trastorno Delirante y un $4.8 \%$ comprendían un conjunto de otros diagnósticos.Asimismo, si tomamos como referencia el conjunto de pacientes ingresados en una unidad psiquiátrica de agudos, la mayoría presenta patología somática asociada. Por ejemplo, según el estudio descriptivo 
mencionado, un $60 \%$ presentaron alguna patología somática asociada, especialmente la hipertensión en un $26.6 \%$, diabetes en un $11.3 \%$ y cardiopatía isquémica el $11.3 \%$.

\section{Hipótesis}

Los pacientes con enfermedad mental y mayores de 65 años ingresados en una unidad psiquiátrica de corta estancia que presenten deterioro cognitivo tienen una prevalencia superior de sintomatología psiquiátrica respecto a los que no presentan deterioro cognitivo.

\section{Objetivos}

Principal: Establecer la relación entre el deterioro cognitivo en personas mayores ingresadas en una Unidad de Corta Estancia Psiquiátrica y las alteraciones psiquiátricas que se producen durante su ingreso.

Secundarios:

Describir el deterioro cognitivo al ingreso de las personas mayores de 65 años que ingresan en un UCE psiquiátrica.

Analizar los SCPD que se producen durante la estancia de los mismos.

Conocer la relación entre el deterioro cognitivo y los SCPD con las variables socio-demográficas recogidas en el estudio.

Relacionar el deterioro cognitivo con el grado de dependencia para las AVD.

\section{METODOLOGÍA}

Diseño: Estudio transversal de prevalencia.

Lugar de realización del proyecto: El marco de referencia en el que se realiza nuestro estudio es el Servicio de Psiquiatría del Hospital San Jorge de Huesca. Se trata de una unidad de corta estancia psiquiatría integrada en el hospital desde hace más de 10 años. Cuenta con 21 camas en las que se atiende de forma aguda a los pacientes psiquiátricos de toda la provincia de Huesca. La provincia de Huesca tiene una población de 228.361 habitantes (a uno de Enero del 2011) de las cuales casi 50.000 son mayores de 65 años.

Población de estudio: La población a estudiar son las personas mayores que ingresen en la unidad de corta estancia de psiquiatría del Hospital San Jorge de Huesca desde el 1 de enero del 2012 hasta el 31 de marzo del 2012.

Criterios de inclusión: Pacientes mayores o iguales a 65 años que ingresen en la unidad de corta estancia de psiquiatría del Hospital San Jorge de Huesca durante el periodo mencionado.

Criterios de exclusión:

Pacientes cuyo tiempo de ingreso sea igual o inferior a 48 horas. 
Pacientes que no afecten el consentimiento informado previo a la recogida de datos para el estudio.

Variables:

Socio-demográficas: edad, sexo, estado civil (soltero, casado y viudo), procedencia (rural o urbana), ámbito (hogar o residencia).

Variables clínica generales: motivo de ingreso, diagnóstico médico principal al alta.

Variables de estructura: número de días de hospitalización.

Variables de valoración funcional: índice de Barthel.

Variables de valoración Cognitiva: Test MEC de Lobo, Test de Pfeiffer y Test de Fluencia Verbal Semántica (animales en 1 minuto).

Variables de valoración psicológica/conductual: Inventario Neuropsiquiátrico de Cummings (NPI).

Recogida de datos: Para la exploración cognitiva y funcional se realizarán mediante la entrevista directa con el paciente el MEC de Lobo, el test de Pfeiffer y a través también de la observación el índice de Barthel. Todo ello en las primeras 48-72 horas del ingreso de los pacientes incluidos en el estudio. Al mismo tiempo se recogerán las variables socio-demográficas descritas con anterioridad.

Al alta del paciente se recogerán los datos necesarios para rellenar el Inventario Neuropsiquiátrico mediante la entrevista con el psiquiatra de referencia durante el ingreso y consultando las anotaciones descritas en las observaciones de enfermería y el informen de continuidad de cuidados enfermeros.

\section{Instrumentos de valoración}

Valoración Cognitiva: Los tests más utilizados para valorar el deterioro cognitivo son el MMSE (Mini Mental Status Examination, de Folstein) y el MEC este último es una versión del anterior, adaptada y traducida a nuestro idioma, y validada en varios estudios (Lobo et al., 1999) que usaremos en esta investigación.

Hay circunstancias en que el MEC (o el MMSE) no se pueden utilizar porque la persona mayor presenta algún déficit sensorial importante, o por analfabetismo. En estos casos, las Guías y Protocolos ofrecen algunas alternativas con la finalidad de objetivar el Déficit Cognitivo que presenta el anciano. Las alternativas más utilizadas son el Short Portable Mental State Questionaire (SPMSQ) de Pfeiffer (Martínez de la Iglesia, Dueñas y Onis, 2001) o el Set Test de Isaac (Pascual-Millán et al., 1990), utilizados ambos en este estudio.

Valoración Funcional: Entre la incapacidad y los trastornos crónicos de salud frecuentes en la vejez, se da una estrecha relación, a veces de carácter circular: los problemas de salud son factores de riesgo de aparición de incapacidad, y la incapacidad es un factor de riesgo de aparición de patologías. 
Uno de los cuestionarios más utilizados para valorar el grado de discapacidad es el índice de Barthel (Cid y Damián, 1997).

Valoración psicológica y conductual: En la actualidad el instrumento de detección de síntomas psicológicos y conductuales en la demencia más utilizado en todo el mundo es el Neuropsychiatric Inventory (NPI) de Cummmings; del cual existe una versión en castellano, denominada Inventario Neuropsiquiátrico (Vitalta-Franch et al., 1999).

Valoración sociodemográfica: edad, sexo, estado civil, motivo de ingreso, diagnóstico médico al alta, domicilio (institución/hogar), medio (rural/urbano).

\section{RESULTADOS}

Durante el periodo de recogida de datos ingresaron 21 pacientes mayores de 65 años que cumplían los criterios de inclusión de los cuales 3 de ellos fueron excluidos por no dar su consentimiento para el estudio. Por tanto se recogieron los datos de 18 pacientes en total.

\section{Variables sociodemográficas}

Los datos sociodemográficos de la muestra se recogen en la siguiente tabla:

Tabla 1. Características de la muestra

\begin{tabular}{cccccccccc}
\hline VARIABLES & \multicolumn{2}{c}{ SEXO } & \multicolumn{3}{c}{ ESTADO CIVIL } & \multicolumn{2}{c}{ PROCEDENCIA } & \multicolumn{2}{c}{ ÁMBITO } \\
\hline Categoría & Hombres & Mujeres & Soltero & Casado & Viudo & Rural & Urbana & Hogar & Residencia \\
\hline$N=18$ & 9 & 9 & 4 & 8 & 6 & 12 & 6 & 13 & 5 \\
\hline$\%$ & 50 & 50 & 22.2 & 44.5 & 33.3 & 66.7 & 33.3 & 72.2 & 27.8 \\
\hline
\end{tabular}

La media de edad de los encuestados fue de 74.89 años, con una desviación típica de 6.49, correspondiendo la cifra de edad más alta la de 90 años. En cuanto a la distribución por sexo fue equitativa (50\%). La mayor parte de la muestra estaba casada (44.4\%), el $33.3 \%$ viudos y el resto solteros.

Dos tercios de la población procedía del ámbito rural y el $72.2 \%$ vivían en su domicilio particular.

\section{Variables clínicas}

En el siguiente diagrama se muestran los diagnósticos psiquiátricos principales recogidos (Gráfica 1). Se observa que destacan la depresión y la depresión psicótica, entre los dos acumulan la mitad de los casos. 
Gráfica 1. Distribución de los diagnósticos principales al ingreso

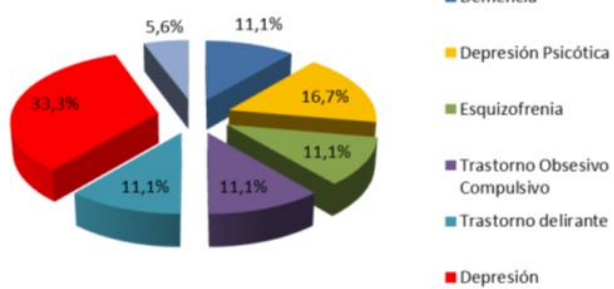

En relación con el motivo de ingreso, también analizado, se desprende que la mitad de los ingresos son provocados por una situación de crisis (entendida como una descompensación imposible de controlar en el entorno del paciente) en su enfermedad metal de base de cada sujeto. También cabe destacan que el suicidio (tanto ideación como el intento autolítico) es la causa de ingreso en el $33.3 \%$ de la población estudiada. (Gráfica 2). La media en cuanto a días de estancia fue de 21.72, con una desviación típica de 13.72 .

Gráfica 2. Distribución por frecuencias de los motivos de ingreso

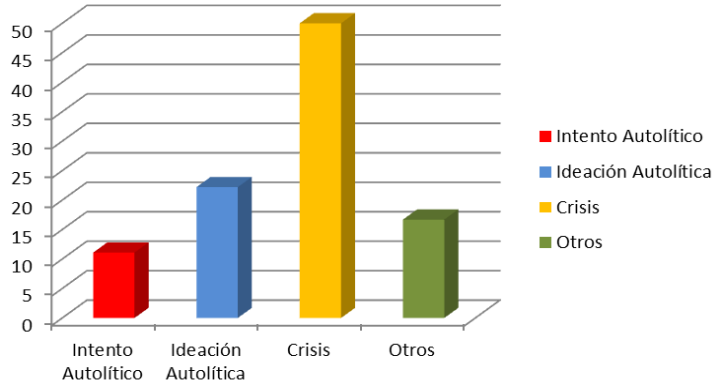

Variables de las encuestas

Se recogieron los datos de 3 test de cribado para el deterioro cognitivo (MEC, Pfeiffer y fluencia verbal) y a su vez del NPI y del Barthel con los siguientes resultados (Tabla 2):

En cuanto al MEC se desprende que el $66.7 \%$ de los pacientes no tiene deterioro cognitivo (punto de corte $<23$ ), con una media de 24.72 .

El los datos del Pfeiffer analizados el punto de corte del deterioro cognitivo se estableció en $<7$ respuestas acertadas, cumpliendo criterios de deterioro cognitivo según este test el $44.4 \%$ de la muestra. La media fue de 6.78, indicativo de deterioro cognitivo. 
La fluencia verbal en animales arrojó una media de 8.83 estableciendo el límite del deterioro cognitivo en una puntuación de 7. El porcentaje del deterioro cognitivo en la muestra fue de $22.2 \%$

El NPI obtuvo una media de 44.33 puntos con una desviación típica de 20.53 y el Barthel un 76.94 y una desviación típica de 22.89.

Al correlacionar los test de cribaje cognitivo empleados en este estudio se obtuvo significación estadística $(\mathrm{p}<0.05)$ entre los tres usados $(\mathrm{MEC}$, Pfeiffer y Fluencia verbal) (Tabla 3).

Tabla 2. Puntuación medias y grado de afectación del deterioro cognitivo

\begin{tabular}{lcccc}
\hline \multirow{2}{*}{ TEST } & \multirow{2}{*}{ MEDIA } & \multirow{2}{*}{ D.T. } & \multicolumn{2}{c}{ GRADO DE DETERIORO $(\%)$} \\
\cline { 4 - 5 } & & & SIN DETERIORO & CON DETERIORO \\
\hline MEC & 24.72 & 8.2 & 66.7 & 33.3 \\
\hline PFEIFFER & 6.78 & 2.81 & 55.6 & 44.4 \\
\hline FLUENCIA & 8.83 & 3.61 & 77.8 & 22.2 \\
\hline
\end{tabular}

Tabla 3. Correlaciones entre las diferentes variables

\begin{tabular}{|c|c|c|c|c|c|c|}
\hline & EDAD & DÍAS DE EST. & MEC & PFEIFFER & FLUENCIA & NPI \\
\hline \multicolumn{7}{|l|}{ EDAD } \\
\hline DIAS DE EST. & 0.58 & & & & & \\
\hline MEC & 0.709 & 0.969 & & & & \\
\hline PFEIFFER & 0.521 & 0.718 & $<0.001$ & & & \\
\hline FLUENCIA & 0.442 & 0.688 & $<0.001$ & $<0.001$ & & \\
\hline NPI & 0.052 & 0.08 & 0.097 & 0.021 & 0.082 & \\
\hline BARTHEL & 0.474 & 0.014 & 0.076 & 0.093 & 0.2 & 0.035 \\
\hline
\end{tabular}

De éste análisis también se desprende que el test de Pfeiffer, que mide el deterioro cognitivo, tiene significación al relacionarlo con las alteraciones psiquiátricas cuantificadas con el NPI y a su vez este test con el índice de Barthel (discapacidad en las AVD).

En cuanto a la edad cabe destacar que aunque no existe significación estadística con el NPI (alteraciones psicológicas y conductuales) su valor de 0.052 es muy próximo.

Con respeto a los días de estancia su relación es relevante con la dependencia en las actividades básicas de la vida diaria (índice de Barthel).

El resto de variables socio-demográficas y clínicas no pueden extraerse conclusiones significativas al ser la muestra muy pequeña.

\section{DISCUSIÓN Y CONCLUSIONES}

El $85.71 \%$ de las pacientes ingresados durante los 3 meses de recogida de los datos participó en el estudio, solamente 3 mostraron su negativa a participar. La alta 
participación puede ser debía a la información tanto oral como escrita recibida previamente a la aceptación de la participación en la investigación (consentimiento informado) o tal vez que la recogida de los datos se realizaba por su cuidador dentro de la unidad psiquiátrica.

La muestra se distribuyó equitativamente respecto al sexo, el $44.5 \%$ estaban casados, el $66.7 \%$ procedían del ámbito rural y el $72.2 \%$ vivían en sus domicilios particulares. Lo que hace presuponer que la mayoría de los sujetos viven acompañados, en el medio rural y en su casa propia, estableciéndose el cuidado dentro de este entramado social.

En el año 2011 se dieron de alta en esta unidad de corta estancia de psiquiatría a 88 personas mayores de 65 años, de un total de 1150 pacientes. La media de estancia de estas personas supuso 13.07 días. Esta media es significativamente menor a la de nuestro estudio (21.72), debido probablemente a que en este estudio se descartaron a los pacientes cuyo ingreso duró menos de 48 horas.

Un tercio de los pacientes analizados ingreso con depresión como diagnóstico principal, y si sumamos la depresión psicótico suponen la mitad de la muestra. De los 18 pacientes 8 ingresaron por una situación de crisis en su patología mental de base. La ideación autolítica fue la causa del ingreso en un tercio de los mayores de 65 años. En este estudio, como ocurre en otros realizados sobre el paciente psicogeriátrico, la población anciana tiene un alto nivel de síntomas depresivos.

En cuanto al deterioro cognitivo los resultados analizados de los test de cribado del mismo concluyen que entre el $22 \%$ y el $44 \%$ (en función del test utilizado) de los pacientes incluidos en la investigación presenta indicios de deterioro cognitivo. Por tanto cabe destacar la importancia de elegir el test a utilizar según el tipo de estudio que se lleve a cabo así como de la población analizada.

Al comparar las alteración psicológicas y conductuales medidas a través del NPI con las test 3 test utilizados para clasificar el deterioro cognitivo, se comprobó que sólo había significación estadística con uno de ellos, el Pfeiffer. El motivo puede ser debido a que este cuestionario tiene menor sensibilidad en pacientes con deterioro más leve, y por tanto cuándo puntúa deterioro es que éste es realmente grave y por ello se asocia más con los síntomas neuropsiquiátricos (SPCD).

Al comparar los test de cribado del deterioro cognitivo (MEC, Pfeiffer y Fluencia Verbal) se obtuvo significación estadística en su concordancia al ser los tres ampliamente validados y ser usados para el mismo propósito de clasificación en el ámbito sanitario.

La puntuación en el NPI y los días de estancia están relacionados con la escala de discapacidad Barthel. Los que supone que a mayor discapacidad en la AVD más días de ingreso hospitalario. Lo mismo ocurre con la NPI, en pacientes más dependientes se producirán mayores alteraciones psiquiátricas. 
Una de las limitaciones de este estudio es el número de pacientes analizados, lo cual disminuye la potencia estadística de los resultados. Con una población mayor se podrían analizar todas las variables recogidas en este estudio, que con el tamaño de muestra obtenido no es posible. También para futuras investigaciones se podría analizar cada una de las áreas por las que está dividido el NPI con las diferentes variables analizadas así como hacer una clasificación más detallada por niveles cognitivos y funcionales de los pacientes.

Otra posibilidad a tener en cuenta en futuras investigaciones sobre este tema sería el comparar las escalas empleadas para la recogida de datos en el momento del ingreso y al alta para hacer una valoración más precisa.

La hipótesis planteada de que los mayores de 65 años con mayor deterioro cognitivo ingresos en una unidad de corta estancia de psiquiatría presentan durante el ingreso mayores alteraciones conductuales y psicológicas se ha demostrado para uno de los test utilizados.

También existe relación entre los días de ingreso y el Barthel así como del Barthel y el NPI lo que supone que controlar las alteraciones psicológicas y conductuales durante el ingreso favorece la independencia en las AVD y con ello se reducen los días de ingreso. También sucede a la inversa, si no controlamos las manifestaciones psiquiátricas habrá más dependencia para las actividades diarias y se alargara el tiempo de estancia. Por tanto realizar cuidados de enfermería encaminados a mitigar o eliminar las 12 áreas de las que se compone el NPI así como aumentar la voluntad, fuerza o conocimiento para favorecer la independencia en las AVD reduciría los días de estancia. Al reducir los días de ingreso mejoramos la calidad de vida de los usuarios sin olvidarnos de la reducción de costes económicos que supone disminuir la estancia media.

Una propuesta de futuro para investigaciones sobre este tema es el planteamiento de un estudio prospectivo durante un año de seguimiento de este tipo de paciente psicogeriátrico en una unidad psiquiátrica de agudos.

\section{REFERENCIAS}

Ballard, C.G., O`Brien, J., James, I. y Swan, A. (2001). Dementia: management of behavioural and psychological symptoms. New York: Oxford University Press.

Blazer, D. (2000). Psychiatry and the oldest old. American Journal of Psychiatry, 157, 1915-1924.

Cid, J. y Damián, J. (1997). Valoración de la discapacidad física: el índice de Barthel. Revista Española de Salud Pública, 71,127-37.

Finkel, S.I., Costa e Silva, J., Cohen, G., Miller, S. y Sartorius, N. (1996). Behavioral and psychological signs and symptoms of dementia: a consensus stamen on current knowledge and implications for research and treatment. International Psychogeriatric, $8(3), 497-500$. 
Lobo, A., Saz, P., Marcos, G., Día, J.L, De la Cámara, C. y Ventura, T. (1999). Revalidación y normalización del Min-Examen-Cognoscitivo en la población general geriátrica. Medicina Clínica, 112, 767-774.

Martínez de la Iglesia, J., Dueñas, R. y Onis, M.C. (2001). Adaptación y valoración al castellano del cuestionario Pfeiffer (SPMSQ) para detectar la existencia de deterioro cognitivo en personas mayores de 65 años. Medicina Clínica, 117, 129-34.

O’Keeffe, S. y Lavan, J. (1997). The prognostic significance of delirium in older hospital patients. Journal of the American Geriatrics Society, 45, 174-178.

Pascual-Millán, L.F., Martínez, J.V., Modrego, P., Mostacero, E., López del Val, L.J. y Morales, F. (1990). El set-test en el diagnóstico de la demencia. Neurología, 5, 82-5.

Subirá, M. y Cardoner, N. (2010). El paciente psicogeriátrico en el Hospital General. Informes psiquiátricas: Publicación científica de los Centros de la Congregación de Hermanas Hospitalarias del Sagrado Corazón de Jesús, 200, 115-122.

Vilalta-Franch, J., Lozano, M., Hernández, M., Llinás, J., López-Pousa, S. y López, O.L. (1999). Neuropsychiatric Inventory: propiedades psicométricas de su adaptación al español. Revista de Neurología, 29(1), 15-19.

Recibido: 22 de abril de 2014 Recepción Modificaciones: 26 de mayo de 2014 Aceptado: 11 de junio de 2014 